\title{
Primary Unreamed and Unlocked Intramedullary Nailing of Femoral Shaft Fractures
}

\author{
EJB Sié, MD Orth, AD Kacou, MD Orth, A Traoré, MD Orth, BL Séry, MD Orth, Y Lambin, MD Orth \\ Department of Orthopedic Surgery, Yopougon University Teaching Hospital, Abidjan, Ivory Coast
}

\begin{abstract}
In this retrospective study, we present our experience using open Küntscher nailing (K-nailing) which is still performed in developing countries for femoral fractures. Of 157 acute fractures treated between January 2003 and December 2009, 100 were stable $(63.7 \%)$ and 135 were located within the middle third of the shaft $(86 \%)$. Comminution was absent or minimal in $135(86 \%)$ cases. Fracture union was achieved at an average of 14 weeks (range, $10-25$ ). The duration of follow-up was 17 months (range, 6 - 36). Final evaluation showed that $129(82.2 \%)$ patients had a good result according to modified Kempf's criteria. The predominant complications were infection $(n=5 ; 3.2 \%)$, nonunion $(n=9$; $5.5 \%)$, implant failure $(n=11 ; 7 \%)$, and malunion $(n=27$; $17.3 \%$ ). Open reduction and fixation with a tight fitting Knail can give good results in selected acute femoral shaft fractures but knowledge of potential complications is needed.
\end{abstract}

Key Words:

femoral shaft fracture, Intramedullary nailing, Open reduction

\section{INTRODUCTION}

Closed locked intramedullary nailing (IM nailing) is the treatment of choice for most femoral shaft fractures (FSFs) ${ }^{1-3}$. This requires use of an image intensifier, a fracture table, appropriate instrumentation, and specialized implants which are not available to most surgeons in developing nations. In resource poor countries, open reduction and internal fixation with K-nails is a readily available and preferred method for treatment of FSFs as it carries the advantages of relatively low cost, short duration of surgery, acceptable intraoperative bleeding in acute fractures, and reasonable clinical outcomes ${ }^{4,5}$. Since the advent of Surgical Implant Generation Network (SIGN) nails, open K-nailing is no longer the common procedure for nailing FSFs ${ }^{6,7}$. This study presents the treatment course and subsequent results of open unreamed and unlocked K-nailing in patients treated for FSFs, with emphasis on surgical errors and complications.

\section{MATERIALS AND METHODS}

Patients treated surgically between January 2003 and December 2009 for FSFs were retrospectively reviewed at the Yopougon University Teaching Hospital, Abidjan, Ivory Coast. Given the local constraints of accessibility and costs, a minimum of 6 months follow-up after discharge was thought to be adequate. For evaluation purposes, the femoral shaft from $5 \mathrm{~cm}$ distal to the lesser trochanter to $5 \mathrm{~cm}$ proximal to the adductor tubercle was divided into three equal thirds ${ }^{4}$. We included all adult (above 15 years old) patients treated initially with open unreamed and unlocked $\mathrm{K}$-nail for isolated acute (less than 4 weeks old $^{8}$ ) fractures. Supracondylar and pertrochanteric fractures, patients treated initially using implants other than K-nail, pathologic fractures, and those in which IM nailing was used as a revision technique to correct previous internal fixation, were excluded. Fractures as a result of gunshot injury were excluded as it is unclear whether the majority of gunshot wounds to the femur can be classified like other open fractures and assigned to categories defined by Gustilo. Polytrauma patients with ipsilateral lower limb fractures or concomitant fractures of the other limbs were also excluded because these injuries later interfere with evaluation of weight bearing and other outcomes. Patients lost to followup and those who died during hospitalization due to causes other than fracture repair or after discharge from hospital were excluded from this study. The initial study population was 192 but 35 patients were excluded because of the above criteria. The final study population was 157 patients with a mean age of 37years (range, $16-67$ ). The average delay from injury to operation was 7days (range, 1-28). Open fractures are classified according to the Gustilo system and comminution as per criteria of Winquist.

The surgical technique has been well described ${ }^{4,5}$. After initial assessment and resuscitation where appropriate, all patients were placed on skin traction if there was likely to be any delay before surgery. Nail diameter was determined by measuring the narrowest medullary diameter normally at the isthmus (just proximal to the middle of the femur).

Open fractures were debrided and fixed in the same setting. All patients were placed in lateral position and operated on 
under general anaesthesia. A posterolateral approach was used to reach the fracture fragments, and these were cleaned with minimum periosteal stripping.

A trochar end guide pin was introduced retrogade until it came out at the area of the piriform fossa and tented into the skin. An incision was made over the tenting guide pin, and the skin and the soft tissues were reflected down to the tip of the greater trochanter.

A K-nail with the appropriate length was introduced retrograde through the proximal fragment. The fracture was then anatomically reduced under direct vision and the nail inserted into the distal fragment by punching. Rotation was corrected using the linea aspera as a marker. The nail was left protruding $2 \mathrm{~cm}$ proximal to the greater trochanter to facilitate later removal. The diameter of nails used was 10 or $11 \mathrm{~mm}(\mathrm{n}=17 ; 10.8 \%), 12$ or $13 \mathrm{~mm}(\mathrm{n}=90 ; 57.3 \%)$, and 14 or $15 \mathrm{~mm}(\mathrm{n}=43 ; 27.4 \%)$. The length of nails inserted was between 34 and $40 \mathrm{~cm} \mathrm{(n=90;57.3 \% ),} \mathrm{or} 41$ and $45 \mathrm{~cm}$ $(n=67: 42.7 \%)$. Cerclage wire was used in addition to the nailing in 20 cases to correct oblique long, spiroid, and cortical splitting that occurred during the nailing procedure. The wound was closed routinely over a suction drain. The knee was passively manipulated through a full range of motion after fracture repair. Postoperatively, a leg cast with a de-rotation device was applied in all cases for 8 weeks to refrain from premature weight bearing. The patients were encouraged to do quadriceps exercises as tolerated.

The time to weight bearing was determined on the basis of the fracture stability and healing progression. There was no specified protocol for routine follow-up.

In general, patients were seen every two weeks for a minimum of eight weeks postoperatively and monthly thereafter. We were mainly concerned about perioperative complications, wound infection, implant failures, union, and similar related complications. Fracture union was defined as absence of pain on full weight bearing and bridging callus on both anteroposterior and lateral radiographic views ${ }^{4}$.

Fractures taking longer than 6 months to unite were defined as delayed union while an absence of callus at the fracture site at 6 months was considered nonunion ${ }^{9}$. Malunion was considered as deformity more than $10^{\circ}$, malrotation alignment more than $10^{\circ}$, shortening more than $2 \mathrm{~cm}$, or any combination thereof ${ }^{3,10}$. Angular deformity was determined by examining radiographs in both anteroposterior and lateral views. Limb shortening was evaluated with the patient supine and the pelvis squared. A tape measure was used to measure the difference in distance between the anterior superior iliac spine and the medial malleolus ${ }^{11}$. Rotational alignment was not consistently recorded in the charts, thus we did not measure this deformity. For the final assessment, the patients were evaluated according to modified Kempf's criteria $^{3}$. After the index operation, a satisfactory result was defined by uneventful wound healing, a fracture union in good position according the aforementioned criteria without pain, within identified delay times, and flexion of the knee of at least $90^{\circ}$. A patient who did not meet these criteria was considered to have a complication. Each complication was counted as occurring in isolation even if the patient experienced several complications.

\section{RESULTS}

The main patient demographics and fracture characteristics are summarized in Table I. The mean duration of operation was 60 minutes (range, 45-150). The average postoperative hospital stay was 8 days (range, 6- 32). The mean delay for uneventful wound healing was 17 days (range, 14- 23). The duration of follow-up was 17 months (range, 6-36). Fracture union was achieved at an average of 14 weeks (range, 1025). Removal of nail was carried out in 37 cases after fracture healing. In 12 cases this was done for hip pain, and in the remainder at patient request. On average, the nails were removed 15 months after insertion. At follow-up, satisfactory results were achieved in $129(82.2 \%)$ patients after the index operation. There were three $(1.9 \%)$ deep vein thrombosis events; all were treated successfully with an appropriate adjustment of low molecular weight heparin dosage. Cortical spitting at the fracture site was seen in 7 $(4.5 \%)$ patients. Additional cerclage wire was successfully used in these patients. Jamming of the nail occurred intraoperatively on $6(3.8 \%)$ occasions, and all were extracted. Five $(3.2 \%)$ patients had wound hematoma that responded to wound care and antibiotics. Five (3.2\%) nails were observed to be too long proximally on the first postoperative radiographs. Three patients required reimpaction of the nail. In two patients, the nails were withdrawn using the incision over the greater trochanter. Nails of correct length were then inserted over a guide wire. Three $(1.9 \%)$ nails protruding into the knee joint were removed and K-nails of correct length were inserted over the guide. Iatrogenic non-displaced femoral neck fractures occurred in $3(1.9 \%)$ cases; this fracture was at first misdiagnosed in one patient. Since fractures were not displaced, a spica cast was applied in all cases and weight bearing was delayed for five months. Fracture healing of the neck was demonstrated in two patients while nonunion occurred in one.

Wound infection was observed in five $(3.2 \%)$ patients, of whom three sustained an open fracture. Infection was superficial in two cases and treated successfully with antibiotics and wound care. The three deep infections occurred two weeks after the operation and were treated with early debridement, drainage, and parenteral antibiotics. The isolated organisms were Pseudomonas aeruginosa in two cases and Staphylococcus aureus in one. Infection had resolved in one patient. Bone union was achieved in one patient but chronic osteomyelitis developed necessitating removal of the nail combined with sequestrectomy. In one patient deep infection did not resolve, and septic nonunion ensued; bone union and wound healing were achieved using a staged protocol. 
Table I: Patient's demographics and fracture characteristics

\begin{tabular}{|lc|}
\hline Parameters & Number of subjects \\
\hline Sex & \\
Male & 112 \\
Female & 45 \\
Mechanisms & 138 \\
Road traffic accident & 13 \\
Fall & 6 \\
Assault & 13 \\
Location of fracture & 135 \\
Proximal third & 9 \\
Middle third & \\
Distal third & 69 \\
Pattern of fracture & 31 \\
Transverse & 7 \\
Short oblique & 11 \\
Oblique long & 20 \\
Spiral & 12 \\
Butterfly & 7 \\
Comminuted & \\
Segmental & 118 \\
Fracture comminution & 17 \\
Type I & 12 \\
Type II & 10 \\
Type III & 133 \\
Type IV & 24 \\
Closed fractures & 10 \\
Open fractures & 9 \\
Type I & 5 \\
Type II & \\
Type III & \\
& \\
\hline
\end{tabular}

In $8(6 \%)$ patients, a bent $\mathrm{K}$-nail was noted three months after surgery; in these cases, the nail diameter was $10 \mathrm{~mm}$ $(\mathrm{n}=5), 11 \mathrm{~mm}(\mathrm{n}=2)$, and $12 \mathrm{~mm}(\mathrm{n}=1)$. These fractures were located in the proximal third in 5 patients, middle in two and the distal in one. In one patient with diameter of $12 \mathrm{~mm}$ and one patient with $11 \mathrm{~mm}$, weight bearing was delayed since the varus deformity was $5^{\circ}$. Bone union was achieved two months later. In the other patients, reoperation was performed since the nails were small and the deformity was $10^{\circ}$. Re-operation consisted of plate osteosynthesis and bone grafting. Fracture union was achieved in four patients three months after the second operation. In one patient, delayed union was noticed after the second operation, while the other one patient went on to aseptic nonunion.

Nail breakage was noted at the fracture site in three $(2 \%)$ patients. In one of these patients, fracture union had already occurred. The proximal part of the nail was removed 16 months after the injury. The other two patients experienced this complication two months after the index operation. They were re-operated with exchange of the nail after reaming coupled with bone grafting, resulting in fracture union three months later.

Delayed union was observed in 7 (4.6\%) cases, of which one was re-operated for a bent nail. Full weight bearing was permitted in all patients who went on to union. Nonunion occurred in $9(5.7 \%)$ cases, of which one was septic. Fractures were located in the distal third in 7 cases with gross comminution (Winquist III and IV). Fractures were open in five cases. Patients with nonseptic nonunion underwent exchange IM nailing (in five) after reaming, plate fixation for proximal third fractures (in two), and Judet screw plate for the distal third (in one). Iliac bone grafting was performed in all cases. After a mean period of 11 months post-injury, bone union was documented in six patients. In the patient with septic nonunion, the staged protocol consisted of removal of the nail and sequestrectomy followed by a second phase of nailing and bone grafting.

Shortening more than $2 \mathrm{~cm}$ was noted in $10(6.4 \%)$ cases. In those patients, gross comminution was present in seven patients and cortical splitting had occurred in four. Varus malunion more than $10^{\circ}$ was seen in five $(3.2 \%)$ patients and valgus more than $10^{\circ}$ was noticed in seven $(4.5 \%)$ patients. Knee flexion more than $90^{\circ}$ was recorded in five $(3.2 \%)$ cases.

\section{DISCUSSION}

When it is more than three weeks post-trauma, closed IM nailing is difficult. The main problems are difficulty in obtaining reduction and passing the guide in the presence of fibrous tissue and fibrous callus at the fracture site ${ }^{12}$. Knailing is readily available and less expensive for use in an open reduction even in the setting of fresh fractures. Unlocked IM nailing was initially developed for fixation of transverse and short oblique fractures around the midshaft or the isthmus ${ }^{4,5,13}$. Overall results obtained are comparable to that achieved with standard interlocking nailing ${ }^{9,13}$.

We used unreamed nailing since the complete ranges of reamers were not available. The issue of whether to ream or not is still debated and comparative studies give conflicting results ${ }^{1}$. Selvakumar et $a l^{14}$. working in a setting similar to ours recommended unreamed nails in selected conditions such as polytrauma where a shorter operative time and less blood loss is desired. In the current report, indications were extended to unstable fractures including fractures of the distal and proximal thirds and those with gross comminution. The plaster cast with de-rotation was designed to prevent premature weight bearing. After the index operation, 82.2\% of patients achieved a satisfactory result. The criteria used for the present study can be assessed by any surgeon and are relevant when evaluating the procedures efficacy. The ability to squat is a function of flexion at the knee joint. Knee flexion of at least $90^{\circ}$ enables toilet activities. Opening of the fracture site is associated with development of nonunion and an increased risk of wound infection after nailing ${ }^{15}$. These promising results are overshadowed by preventable complications. 
Our rate of malunion of $17.3 \%$ was comparable with that reported in the literature ranging from 6 to $27 \%$ (shortening, angulation, malrotation) ${ }^{11,16}$ but malrotation was not evaluated. In the series by Bajwa et al $^{5}$ there was no rotatory deformity or shortening and fractures were transverse or short oblique with minimal comminution.

No patient had clinically symptomatic angular or rotatory malunion in the study by Devnani ${ }^{4}$. Fifteen of 32 patients in this series had fractures situated either in the proximal or the distal third of the shaft. Despite this, good anatomical results were achieved in those patients since 11 out of these 15 fractures were stable with six having interdigitations.

Limb length discrepancy can result from comminution or cortical splitting that occurs during nailing. To minimize shortening, it is important to use a nail length measured from the intact femur and not adjust the length based on intraoperative findings because in such cases the femur will often end up short ${ }^{11}$.

Malrotation, a common problem with IM nailing, merits special attention. Its incidence would be higher in closed methods. This deformity was not evaluated in our series as in that by Lin et $a l^{13}$. If malrotation is less than 15 to $20^{\circ}$, then the patient can be reassured that it is unlikely to cause a problem. The patient can disguise this deformity when walking provided the hip has good range of motion ${ }^{4,17}$.

Nonunion is a great concern in IM nailing particularly if an unreamed nail is used. Yu et $a l^{9}$ and Lin et $a l^{13}$ using reamed unlocked K-nails, reported a nonunion rate of $4.6 \%$ and $2.3 \%$ respectively. Our rate of nonunion of $5.7 \%$ is in line with that reported in series using unreamed nailing (range, $0 \%$ to $8 \%)^{14,18}$. Nonunion was attributable to the instability of fractures. Indeed comminuted fractures or fractures toward the ends of the shaft will not be held firmly by a standard nail.

This study also confirms that K-nailing has a higher rate of implant failures including migration, bending, and breakage. These complications are frequently seen with small nails or those which did not reach the cancellous distal femur. Surgeons should proceed more slowly in initiating weight bearing protocols when unlocked IM nail fixation is employed ${ }^{9}$.

Studies by Devnani ${ }^{4}$ and Bajwa et $a l^{5}$ with good anatomical results delineate the relevant factors that should be taken into account in treatment decisions in order to decrease the incidence of mechanical complications frequently observed in the current study: (1) fracture location; (2) fracture line geometry; (3) contact between the proximal and distal fragments (i.e., magnitude of comminution); (4) the presence of interdigitation of the fracture ends; and (5) use of correct nail size and length to ensure secure fixation in the cancellous distal femur.

One of the major risks of open K-nailing is infection. In this study the incidence of infection was $3.2 \%$. This is comparable with others studies. The reported incidence of infection complicating open K-nailing for acute femoral fractures varies between $1.5 \%$ and $5 \%{ }^{4,5,13}$. These rates include superficial and deep infections. Superficial infections cause delayed wound healing. In the series by Devnani ${ }^{4}$ comprising 32 patients treated after a mean period of 12 days, two patients developed infection. This settled within 6 weeks with dressings and antibiotics in the patient with superficial infection. Rates of infection in fresh fractures are rather low when compared with that observed in neglected fractures. Akinyoola et $a l^{8}$ reported an infection rate of $23.6 \%$ (both superficial and deep infections) in a series of 55 fractures operated after a mean delay of 24 weeks. The mini open incision required for plate fixation confers the advantages of less contamination, less tissue manipulation, and decreased risk of infection in fresh fractures ${ }^{6}$.

There were several limitations to the present study. This was a retrospective study with several surgeons performing the procedure. The study was conducted in a teaching hospital where residents performed the majority of operations at night. These factors are source of bias and could explain the numerous errors and complications. The relatively large sample size enabled us to identify potential problems that could be prevented and outcomes that could be improved.

\section{CONCLUSION}

Satisfactory results were achieved in $82.2 \%$ of patients using open reduction and fixation with a tight fitting K-nail for selected acute femoral shaft fractures. This procedure is however associated with infection and mechanical complications. The authors hope that the current study serves as a baseline for certain potential preventable errors and complications, since such benchmarks are often sought by clinical investigators for evaluation of fracture fixation methods. 


\section{REFERENCES}

1. Ricci WM, Gallagher B, Haidukewych GH. Intramedullary nailing of femoral shaft fractures: current concepts. $J$ Am Acad Orthop Surg. 2009; 17: 296-305.

2. Winquist RA. Locked femoral nailing. J Am Acad Orthop Surg. 1993; 1: 95-105.

3. Kempf I, Grosse A, Taglang G, Bernhard L, Moui Y. Locked intramedullary nailing of fresh diaphyseal fractures of femur and tibia. A study of 855 cases. Chirurgie 1991; 117: 478-87. [In French].

4. Devnani AS. Open reamed femoral intramedullary nailing. Revisited. Eastern Journal of Medicine. 2003; 8:7-11.

5. Bajwa AS, Schnaid E, Sweet MEB. Current indications for open Küntscher nailing of femoral shaft fractures. East and Central African Journal of Surgery. 2000; 5: 7-10.

6. Ikpeme I, Ngim N, Udosen A, Onuba O, Enembe O, Bello S. External jig aided intramedullary interlocking nailing of diaphyseal fractures: Experience from a tropical developing centre. Int Orthop. 2011; 35: 107-11.

7. Zirkle LG, Shearer D. SIGN technique for retrograde and anterograde approaches to femur. Tech Orthop. 2009; 24: 247-52.

8. Akinyoola L, Oreikha O, Odunsi O. Open intramedullary nailing of open neglected femoral shaft fractures: indications and outcome. Acta Orthop Belg. 2011; 77: 73-7.

9. Yu CK, Wong HY, Vivek AS, SeTo BC. Unlocked nailing vs. interlocking nailing for Winquist type I and II femoral isthmus fractures. Is there a difference? Mal Orth J. 2008; 2: 23-7.

10. Mukherjee SK, Jain V. Neglected femoral diaphyseal fracture. Clin Orthop. 2005; 431: 72-9.

11. Karapinar L, kaya A, Ozturk H, Altay T, Kayali C. Leg length discrepancies in adult femoral shaft fractures treated with intramedullary nailing. Turkish Journal of Trauma and Emergency Surgery. 2009; 15: 256-61.

12. Abrahan LM, Te CV, Cruz FD. Delayed open intramedullary nailing of femoral shaft fractures. Tech Orthop. 2006; 21:88-98.

13. Lin HT, Ng WM, Kwan MK, Fathi Alzudeen LK. Tay PCM. Use of unlocked intramedullary nailing in Winquist type I and II femoral isthmus fracture. Mal Orth J. 2008; 4: 17-22.

14. Selvakumar K, Saw KY, Fathima M. Comparison between reamed and unreamed nailing of cloed femoral fractures. Med $J$ Malaysia. 2001; 56(D): 24-8.

15. Malik MHA, Harwood P, Diggle P, Khan SA. Factors influencing rates of infection and nonunion in intramedullary nailing. $J$ Bone Joint Surg Br. 2004; 86: 556-60.

16. Harris I, Hatfield A, Donald G, Walton J. Outcome after intramedullary nailing of femoral shaft fractures. ANZ J Surg. 2003; 73 : $387-9$.

17. Kent ME, Arora A, Owen PJ, Khanduia V. Assessment and correction of femoral malrotation following intramedullary nailing of the femur. Acta Orthop Belg. 2010; 76: 580-4.

18. Shepherd LE, Shean CJ, Gelalis ID, Lee J, Carter VS. Prospective randomized study of reamed versus unreamed femoral intramedullary naling: An assessment of procedures. J Orthop Trauma. 2001; 15: 28-33. 